\title{
A circulating tumor cell cluster-based model for tumor metastasis (Hypothesis)
}

\author{
YUPENG HONG ${ }^{1},{\mathrm{ZIDUO} \mathrm{LI}^{2} \text { and QI ZHANG }}^{3}$ \\ ${ }^{1}$ Department of Oncology, Zhejiang Provincial People's Hospital, Hangzhou, Zhejiang 310006, P.R. China; \\ ${ }^{2}$ Australian and New Zealand Army Corps Research Institute, University of Sydney, Sydney, New South Wales 2006 , \\ Australia; ${ }^{3}$ Department of Hepatobiliary and Pancreatic Surgery, \\ The Second Affiliated Hospital of Zhejiang University School of Medicine, Hangzhou, Zhejiang 310009, P.R. China
}

Received June 12, 2015; Accepted October 11, 2016

DOI: $10.3892 / \mathrm{ol} .2016 .5358$

\begin{abstract}
Metastasis is the major cause of mortality in patients with malignancies; however, the mechanisms of tumor cell dissemination and metastasis formation are obscure. Circulating tumor cells (CTCs) are believed to be a critical step for distant metastasis and are associated with a poor patient prognosis. The precise processes of metastasis formation from CTCs are vague. In the present study, we hypothesize that two CTC cluster-based mechanisms of tumor cell inoculation in ectopic organs may be viable: i) Formation of a micro-cancer embolus due to interception of CTC clusters by small vessels; and ii) formation of micrometastasis in an extravasation-dependent or -independent manner. Pathological evidence of micro-cancer emboli is critical for the verifications of this hypothesis. If proved true, this hypothesis will provide a novel perspective for cancer metastasis and has valuable clinical implications.
\end{abstract}

\section{Contents}

1. Introduction

2. Hypothesis

3. Test of hypothesis

4. Summary and perspectives

5. Clinical implications

Correspondence to: Dr Qi Zhang, Department of Hepatobiliary and Pancreatic Surgery, The Second Affiliated Hospital of Zhejiang University School of Medicine, 88 Jiefang Road, Hangzhou, Zhejiang 310009, P.R. China

E-mail: zhangqi86@gmail.com

Abbreviations: CTC, circulating tumor cell; EMT, epithelial-mesenchymal transition; EpCAM, epithelial cell adhesion molecule; CSC, cancer stem cell

Key words: CTC cluster, epithelial-mesenchymal transition, metastasis, extravasation, micro-cancer embolus

\section{Introduction}

Circulating tumor cells (CTCs) refer to tumor cells existing in the circulatory system, and they have been increasingly investigated recently due to the belief of their responsibility for tumor metastasis (1). Tumor metastasis is the major cause of mortality in patients with malignancies (2). Although the mechanisms of tumor metastasis are largely unknown, CTCs were considered crucial for tumor metastasis rather than local advancement (3), and in particular, certain subgroups of CTCs can initiate metastases in ectopic organs (4). Several steps were artificially classified for the purpose of a clear understanding of tumor metastasis $(5,6)$, that is, invasion into the circulation system, survival and shift in the circulating system, and extravasation into ectopic organs. There are a number of hypotheses regarding each step of tumor metastasis respectively (7), although none of them have been able to elucidate the whole processes of metastasis.

CellSearch ${ }^{\circledR}$ is now the only certificated method for CTC detection approved by the US Food and Drug Administration, and epithelial cell adhesion molecule (EpCAM) is the most widely used biomarker of CTCs in a variety of methods for CTC detection. However, the validity of EpCAM as the CTC biomarker has been challenged by the identification of CTCs lacking epithelial characteristics $(8,9)$. Epithelial-mesenchymal transition (EMT) has also been noted in CTCs (10), indicating the heterogeneity of the cells. EMT and its reversal process, mesenchymal-epithelial transition, were considered necessary for tumor metastasis $(11,12)$. However, the status of post-EMT tumor cells in the circulatory system is vague and requires further investigation to elucidate the EMT-based metastatic hypotheses.

Notably, CTCs can be found in the peripheral blood in the early stages of numerous malignancies (13-15), while metastasis is considered as concrete evidence of late-stage cancer. This discrepancy suggests that the formation of macrometastasis led by CTC is difficult, even though the critical role of CTCs in tumor metastasis has been widely accepted. On the other hand, persistent release of tumor cells must exist and a CTC pool in the circulatory system is progressively established during tumor progression. The CTC pool implies that the CTCs have a long-term effect on tumor cell dissemination 
and an indeterminable boundary for solid tumors, even those with an intact capsule.

The imperfection of all existing models of tumor metastasis is the mechanism of CTC extravasation and its inducers [such as $\mathrm{C}-\mathrm{X}-\mathrm{C}$ motif chemokine ligand $12, \mathrm{C}-\mathrm{C}$ motif chemokine ligand 2, vascular endothelial growth factor and transforming growth factor- $\beta$ (TGF- $\beta$ )]. Since tumor cells undergoing EMT have a better capacity for invasion and migration, EMT is believed to be a crucial event during extravasation (16). However, it has not been clearly elucidated as to whether EMT is indispensable and the inducer of EMT.

The sites of CTC extravasation are also confusing. Although certain cancer types have preferable metastatic organs (17), non-specific organ metastasis also contributes to considerable cases. The lungs are one of the organs with a high incidence of metastasis from other locations, and the majority of metastases locate in the outer regions of the lungs. A lower bloodstream velocity and a thinner blood vessel diameter are possible reasons for this. Actually, CTCs are bigger in size than the majority of blood cells (18), and their interception by vessels can be observed in vivo (19).

\section{Hypothesis}

We hypothesize that i) extravasation of CTC clusters and single CTCs from vessels induced by interception in relatively small vessels and ii) extravasation-dependent and -independent mechanisms are of importance for tumor metastasis.

A CTC cluster-based model. Multicellular CTC clusters have been identified $(18,20)$, with up to 100 tumor cells found in one cluster (21). Currently, only a few studies have reported the successful detection of CTC clusters, while single CTCs have been universally found in corresponding studies (20-22). We believe that the amount of CTC clusters is largely underestimated by current detection methods, as the majority of these methods use a strategy of surface protein-targeted antibody-based capture. Since the ratio of the superficial area to the volume decreases as the volume increases, the efficiency of CTC capture by antibody-targeting surface proteins is greatly reduced for CTC clusters compared with a single CTC. Therefore, the actual amount of CTC clusters in a patient is underestimated, and the role of CTC clusters in tumor metastasis is largely unknown. CTC detection methods applying novel principles may improve the efficacy of CTC cluster capture and shed light on an in-depth understanding of CTCs in tumor metastasis.

The origin of CTC clusters remains elusive. At present, there are at least three possible origins (Fig. 1). The first is that CTC clusters directly break off from the primary tumor as a result of the shearing force of the blood. Tumor emboli can often be found in patients with late-stage cancer. Solid tumors tend to invade vessels to obtain abundant oxygen and nutrients for cell metabolism and proliferation-associated biosynthesis. Clinical studies have confirmed that patients with vessel invasion by tumors had an increased risk of recurrence and a poorer prognosis compared with those who had tumor-free vessels in a range of cancers, including breast cancer, hepatocellular carcinoma and papillary thyroid carcinoma (23-25). In addition, in patients without pre-operative metastasis, resection of the tumor-invaded vessels conferred a survival benefit, with a similar long-term prognosis compared with patients without vessel invasion by tumors $(26,27)$. Thus, removal of tumor emboli and tumor-invaded vessels can eliminate a critical origin of CTCs and minimize the possibility and extent of tumor metastasis, as well as tumor-associated mortality.

The second origin of CTC clusters can be the result of single CTC proliferation. Although there is a lack of strong evidence regarding the potential of CTCs to resist immune clearance, CTCs were previously found to be associated with impaired immune function in patients $(28,29)$. In addition, certain single CTCs can survive for a considerable time in the circulatory system due to their probable potential of passing through capillaries and proliferating to form CTC clusters. Thus, for certain single CTCs, this process may continue until a big enough CTC cluster has been established and intercepted by vessels with small diameters.

The third origin of CTC clusters is the aggregation of single CTCs (30). Although it is difficult to monitor the formation of CTC clusters by single CTCs in real time in vivo, existing evidence may indirectly support this idea. For instance, normal cells are programmed to die if they are detached from neighboring cells and the extracellular matrix, this is known as anoikis (31). The proportion of non-apoptotic CTCs is essential to the formation of metastases (13). However, tumor cells resist anoikis partially by forming cell clusters $(32,33)$. Notably, characteristics of EMT, including upregulation of vimentin expression and downregulation of E-cadherin expression, were found to be associated with anoikis $(34,35)$. Meanwhile, EMT was also shown to play an important role in CTC cluster formation (21). These complicated phenomena suggest that CTC cluster formation may be a strategy adopted by tumor cells to resist anoikis. On the other hand, it should also be noted that the existence of CTCs is significantly associated with vessel thrombosis (36), which leads to embolism or thrombosis, particularly in microvessels. The formation and special biological characteristics of CTCs have been proven to be responsible for the hypercoagulability and subsequent thrombosis of patients (36); however, the unique physical trait of being cell cluster is also logically associated with a difficulty in passing through the capillaries. In addition, thrombosis and thrombolysis in microvessels can frequently occur in patients with hypercoagulability. Ongoing thrombosis can induce stenosis of vessel lumina, which tends to intercept CTC clusters. Numerous changes occur under these conditions. Embolism or complete thrombosis in microvessels can cause local hypoxia, which has been proven to induce EMT in various cancers, including hepatocellular carcinoma, gastric cancer, pancreatic cancer, breast cancer and colon cancer (37-41). EMT of CTCs may contribute to tumor cell extravasation from vessels into tissues. This model does not require CTCs to permanently sustain mesenchymal characteristics in the circulatory system, and fits well with the fact that the majority of CTCs detected show epithelial characteristics. In addition, the marked change in the inflammation-immune-cytokine local environment induced by platelet aggregation and subsequent cascades can also upregulate certain EMT-triggering factors, such as TGF- $\beta$ (21). These changes provide an initial impetus for CTCs to extravasate from vessels into tissues. 


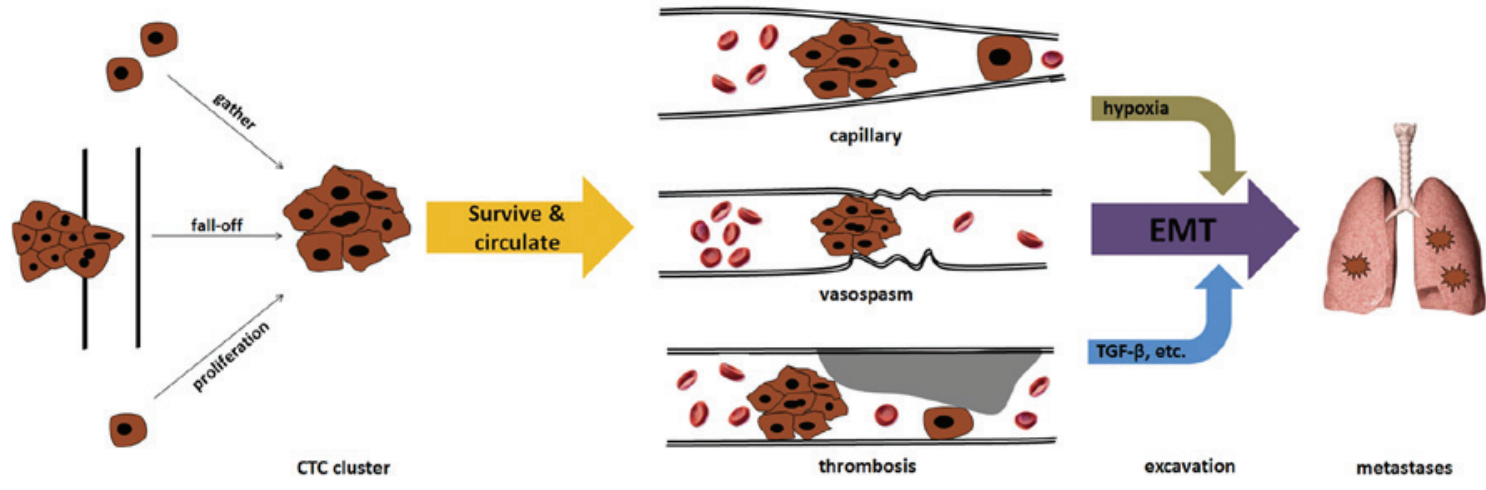

Figure 1. Formation of tumor microemboli. CTC clusters in vessels can originate from a proliferation or gathering of single CTCs, or from direct fall-off from tumor embolization. CTC clusters are prone to be intercepted and form micro-tumor emboli in capillaries, vessels undergoing vasospasm or vessels with thrombosis. Hypoxia caused by micro-tumor embolization or/and TGF- $\beta$ released by platelets can induce the EMT of tumor cells, enhancing their invasion capacity and resulting in extravasation to ectopic organs (e.g., the lungs). CTC, circulating tumor cell; TGF- $\beta$, transforming growth factor- $\beta$; EMT, epithelial-mesenchymal transition.

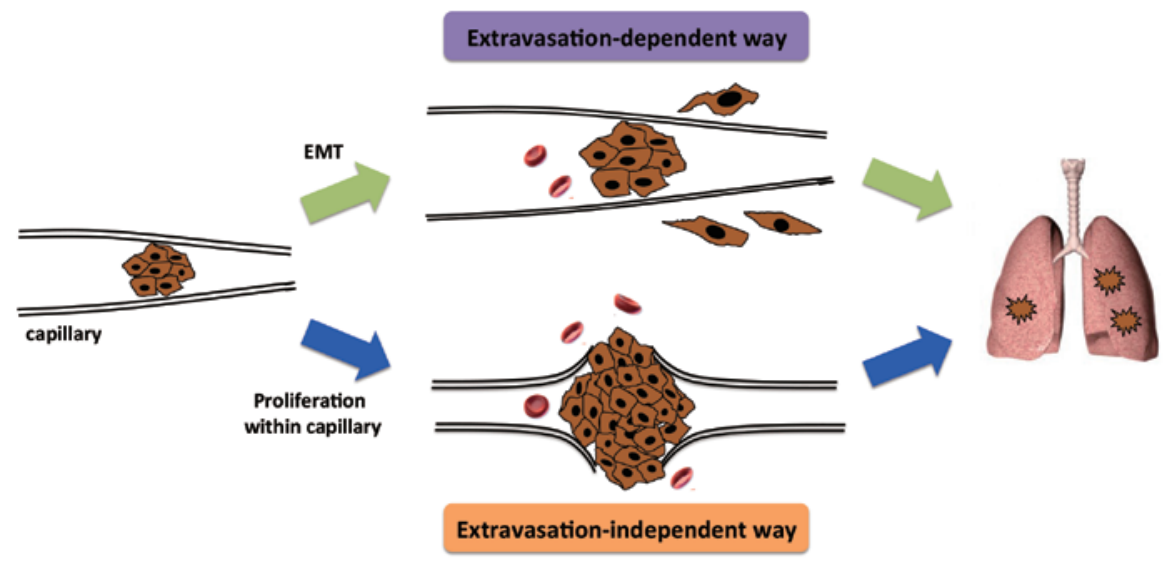

Figure 2. Formation of micrometastases. After interception by small vessels, CTC clusters may undergo EMT and transverse the vascular endothelial cells without damaging the vessel wall. Alternatively, CTC clusters may expand due to the proliferation of cancer cells, resulting in rupture of the vessel. Without undergoing extravasation, the cancer cells can invade into tissues and form micrometastases. CTC, circulating tumor cell; EMT, epithelial-mesenchymal transition.

CTC clusters may have different characteristics according to their origins. For example, CTC clusters directly from the primary tumor may contain evidently more epithelial traits (if the primary tumor is an epithelial malignancy), while those aggregated by single CTCs may tend to be mesenchymal. In addition, heterogeneity may exist within a CTC cluster if it contains a large number of cells. Little is known about the physical or biochemical details of these CTC clusters, including the oxygen and nutrient supply, and the distribution (42).

Single CTC model. To date, the majority of detected CTCs have been single cells and their existence has been reported to be associated with tumor metastasis, treatment response and the long-term prognosis of patients (43-45). However, only certain patients with malignancies exhibited CTCs in their peripheral blood (46). Even in patients with detectable metastases, CTCs could only be detected in half of them (47). The threshold of positive and negative results of CTC detection by successfully detecting one or two CTCs in a $7.5-\mathrm{ml}$ blood sample is also non-robust. Considering the discrepancy of detection of single CTCs (early stage) and tumor metastasis (late stage) regarding the stage of the disease, certain single CTCs are likely to survive in the circulatory system for a long time or alternatively undergo dormancy in tissues or organs (6). Although few CTCs can stay for a long time in the blood (6), the survival and proliferation of CTCs in ectopic organs demands certain necessary prerequisites. Based on the present point of view, abundant cells are prerequisites of tumor inoculation in ectopic organs. Recently, it was demonstrated that the CTC clusters had 23- to 50-fold increased metastatic potential compared with single CTC cells (48).

Cancer stem cells (CSCs), which have a potent capacity for tumor initiation, require a certain number of cells to form tumors subcutaneously in nude mice (49). Although CTCs have a number of the characteristics of CSCs, a certain number of CTCs is also necessary for the formation of tumor metastases (50). From this point of view, single CTCs can barely effectively generate tumor metastases due to a lack of enough cells. A small number of tumor cells in ectopic organs are easily removed by the local immune system or die due to insufficient self-support in the early stage of metastasis (51). While it is impossible that hundreds or thousands of single CTCs independently extravasate from the same anatomical 
site of vessels and aggregate together in ectopic organs, the role of CTC clusters, rather than single CTCs, provides a more logical and rational explanation for the initial formation of tumor metastasis.

Indispensability of dose extravasation. Following interception of CTC clusters, the local microenvironment is relatively stable. Removing the bloodstream and re-attaching to the vascular endothelial cells makes proliferation possible. Expansion of CTC clusters due to cancer cell proliferation can result in the rupture of fragile microvessels, allowing tumor cells to invade into the tissues (Fig. 2). This extravasation-independent manner of micrometastasis formation may be more likely to be associated with the previously mentioned CTC cluster-based metastasis.

\section{Test of hypothesis}

To test our hypothesis, three points are indispensable, namely, histological evidence of micro-cancer emboli, extravasation of the cancer cells from the cancer emboli or rupture of vessels at the site of the cancer emboli, and formation of metastases from these cancer cells.

Since the majority of distant metastasis involves the lungs, liver, bone marrow and brain, lung metastasis can be an ideal model due to convenient sampling and easy observation. Spontaneous tumor models in animals are required, such as metastatic melanoma inoculation in immunodeficient mice. Specimens of lung with hematoxylin and eosin staining, and immunohistological staining with suitable markers should be checked with great care and patience at different times post-inoculation. Genetic or specific protein information can be useful for the identification of cells in a micro-tumor embolus. Alternatively, caudal vein injection of tumor cells is another model for CTC study; however, this model differs from the previously mentioned spontaneous tumor models in that it cannot mimic intravasation of the tumor cells and it has a much simpler intercellular cooperation. Furthermore, single cells or small cell clusters near narrow vessels have to be found. To accomplish this, cell lines expressing green fluorescent protein or other fluorescent proteins are of use to increase the likelihood of identification. Finally, simultaneous evidence of lung metastases and micro-cancer emboli with an identical cell origin are required to verify our hypothesis.

\section{Summary and perspectives}

Based on current evidence, we hypothesize that CTC clusters, whatever their origin, are directly responsible for tumor metastasis, with the stipulation that CTCs can be found in circulatory system. Novel detection and sorting methods for CTC clusters are urgently required to manifest the actual role of CTC clusters in tumor metastasis. Physical techniques based on size, adhesion and electrophysiological characteristics of CTCs may be useful in developing the next generation CTC detection methods, and certain studies have shown promising techniques with significantly increased efficiency of CTC capture (52-54). Furthermore, cell cluster filtration and fractal structure capture systems may improve the efficiency of CTC capture. These strategies cannot only independently increase yield or/and purity of captured CTCs, but also improve the efficiency and specificity of CTC capture synergistically together with novel biomarkers of CTCs. However, there are numerous questions that require answering, such as: i) whether CTC clusters grow/decade or are dynamically self-retained when they moves in the circulatory system; ii) whether the number, proportion, size or nature of CTC clusters is associated with tumor metastasis; iii) whether tumor cells within CTC clusters evolve or undergo EMT; iv) whether there are any specific characteristics of CTC clusters regarding the interaction with the immune system; whether there are any differences in tumor cell extravasation induced by CTC clusters or single CTCs; and vi) what the time-spectrum is of CTC clusters during primary tumor progression according to next-generation detection methods; particularly, whether CTC clusters can be found in the early stages of disease.

By resolving these questions, a further understanding of the characteristics of CTC clusters and their role in tumor metastasis, as well as the integrated mechanism of tumor metastasis, should be gained.

\section{Clinical implications}

The test of these models will provide a novel perspective for cancer metastasis, and assist in improving CTC-based cancer diagnostics and monitoring. Novel strategies for the prevention of cancer metastasis may be also expected once proof has been obtained for these models.

\section{Acknowledgements}

This study was financially supported by the National Natural Science Foundation of China (grant no. 81401954) and the Medical Science and Technology Program of Zhejiang Province, China (grant no. 2015KYA114).

\section{References}

1. Plaks V, Koopman CD and Werb Z: Cancer. Circulating tumor cells. Science 341: 1186-8, 2013.

2. Chaffer CL and Weinberg RA: A perspective on cancer cell metastasis. Science 331: 1559-1564, 2011.

3. Maheswaran S and Haber DA: Circulating tumor cells: A window into cancer biology and metastasis. Curr Opin Genet Dev 20: 96-99, 2010.

4. Baccelli I, Schneeweiss A, Riethdorf S, Stenzinger A, Schillert A, Vogel V, Klein C, Saini M, Bäuerle T, Wallwiener M, et al: Identification of a population of blood circulating tumor cells from breast cancer patients that initiates metastasis in a xenograft assay. Nat Biotechnol 31: 539-544, 2013.

5. Fidler IJ: The pathogenesis of cancer metastasis: The 'seed and soil' hypothesis revisited. Nat Rev Cancer 3: 453-458, 2003.

6. Giancotti FG: Mechanisms governing metastatic dormancy and reactivation. Cell 155: 750-764, 2013.

7. Ramis-Conde I, Chaplain MA, Anderson AR and Drasdo D: Multi-scale modelling of cancer cell intravasation: The role of cadherins in metastasis. Phys Biol 6: 016008, 2009.

8. Gorges TM, Tinhofer I, Drosch M, Röse L, Zollner TM, Krahn T and von Ahsen O: Circulating tumour cells escape from EpCAM-based detection due to epithelial-to-mesenchymal transition. BMC Cancer 12: 178, 2012.

9. Königsberg R, Obermayr E, Bises G, Pfeiler G, Gneist M, Wrba F, de Santis M, Zeillinger R, Hudec M and Dittrich C: Detection of EpCAM positive and negative circulating tumor cells in metastatic breast cancer patients. Acta Oncol 50: 700-710, 2011. 
10. Barrière G, Tartary $M$ and Rigaud $M$ : Epithelial mesenchymal transition: A new insight into the detection of circulating tumor cells. ISRN Oncol 2012: 382010, 2012.

11. Ocaña OH, Córcoles R, Fabra A, et al: Metastatic colonization requires the repression of the epithelial-mesenchymal transition inducer Prrx1. Cancer Cell 22: 709-724, 2012.

12. Tsai JH, Donaher JL, Murphy DA, Chau S and Yang J: Spatiotemporal regulation of epithelial-mesenchymal transition is essential for squamous cell carcinoma metastasis. Cancer Cell 22: 725-736, 2012.

13. Kallergi G, Konstantinidis G, Markomanolaki H, Papadaki MA, Mavroudis D, Stournaras C, Georgoulias V and Agelaki S: Apoptotic circulating tumor cells in early and metastatic breast cancer patients. Mol Cancer Ther 12: 1886-1895, 2013.

14. Nakagawa T, Martinez SR, Goto Y, et al Detection of circulating tumor cells in early-stage breast cancer metastasis to axillary lymph nodes. Clin Cancer Res 13: 4105-4110, 2007.

15. Nagrath S, Sequist LV, Maheswaran S, Bell DW, Irimia D, Ulkus L, Smith MR, Kwak EL, Digumarthy S, Muzikansky A, et al: Isolation of rare circulating tumour cells in cancer patients by microchip technology. Nature 450: 1235-1239, 2007.

16. Wu Y and Zhou BP: New insights of epithelial-mesenchymal transition in cancer metastasis. Acta Biochim Biophys Sin (Shanghai) 40: 643-650, 2008.

17. Nguyen DX, Bos PD and Massagué J: Metastasis: From dissemination to organ-specific colonization. Nat Rev Cancer 9: 274-284, 2009.

18. Yu M, Stott S, Toner M, Maheswaran S and Haber DA: Circulating tumor cells: Approaches to isolation and characterization. J Cell Biol 192: 373-382, 2011.

19. Schlüter K, Gassmann P, Enns A, et al: Organ-specific metastatic tumor cell adhesion and extravasation of colon carcinoma cells with different metastatic potential. Am J Pathol 169: 1064-1073, 2006.

20. Hou HW, Warkiani ME, Khoo BL, Li ZR, Soo RA, Tan DS, Lim WT, Han J, Bhagat AA and Lim CT: Isolation and retrieval of circulating tumor cells using centrifugal forces. Sci Rep 3: 1259, 2013

21. Yu M, Bardia A, Wittner BS, Stott SL, Smas ME, Ting DT, Isakoff SJ, Ciciliano JC, Wells MN, Shah AM, et al: Circulating breast tumor cells exhibit dynamic changes in epithelial and mesenchymal composition. Science 339: 580-584, 2013.

22. Molnar B, Ladanyi A, Tanko L, Sréter L and Tulassay Z: Circulating tumor cell clusters in the peripheral blood of colorectal cancer patients. Clin Cancer Res 7: 4080-4085, 2001.

23. Cianfrocca M and Goldstein LJ: Prognostic and predictive factors in early-stage breast cancer. Oncologist 9: 606-616, 2004

24. Jonas S, Bechstein WO, Steinmüller T, Herrmann M, Radke C, Berg T, Settmacher U and Neuhaus P: Vascular invasion and histopathologic grading determine outcome after liver transplantation for hepatocellular carcinoma in cirrhosis. Hepatology 33: 1080-1086, 2001

25. Falvo L, Catania A, D'Andrea V, Marzullo A, Giustiniani MC and De Antoni E: Prognostic importance of histologic vascular invasion in papillary thyroid carcinoma. Ann Surg 241: 640-646, 2005.

26. Nakagohri T, Kinoshita T, Konishi M, Inoue K and Takahashi S: Survival benefits of portal vein resection for pancreatic cancer. Am J Surg 186: 149-153, 2003.

27. Adham M, Mirza DF, Chapuis F, Mayer AD, Bramhall SR, Coldham C, Baulieux J and Buckels J: Results of vascular resections during pancreatectomy from two European centres: An analysis of survival and disease-free survival explicative factors. HPB (Oxford) 8: 465-473, 2006.

28. Green TL, Cruse JM, Lewis RE and Craft BS: Circulating tumor cells (CTCs) from metastatic breast cancer patients linked to decreased immune function and response to treatment. Exp Mol Pathol 95: 174-179, 2013.

29. Gruber I, Landenberger N, Staebler A, Hahn M, Wallwiener D and Fehm T: Relationship between circulating tumor cells and peripheral T-cells in patients with primary breast cancer. Anticancer Res 33: 2233-2238, 2013.

30. Williams SC: Circulating tumor cells. Proc Natl Acad Sci USA 110: 4861, 2013

31. Frisch SM and Screaton RA: Anoikis mechanisms. Curr Opin Cell Biol 13: 555-562, 2001.

32. Liotta LA and Kohn E: Anoikis: Cancer and the homeless cell. Nature 430: 973-974, 2004

33. Zhang $\mathrm{X}, \mathrm{Xu} \mathrm{LH}$ and $\mathrm{Yu} \mathrm{Q}$ : Cell aggregation induces phosphorylation of PECAM-1 and Pyk2 and promotes tumor cell anchorage-independent growth. Mol Cancer 9: 7, 2010.

34. Kumar S, Park SH, Cieply B, et al: A pathway for the control of anoikis sensitivity by E-cadherin and epithelial-to-mesenchymal transition. Mol Cell Biol 31: 4036-4051, 2011.
35. Xiao Q, Qu K, Wang C, Kong Y, Liu C, Jiang D, Saiyin H, Jia F, $\mathrm{Ni} C$, Chen T, et al: HDGF-related protein-3 is required for anchorage-independent survival and chemoresistance in hepatocellular carcinomas. Gut 62: 440-451, 2013.

36. Tormoen GW, Haley KM, Levine RL and McCarty OJ: Do circulating tumor cells play a role in coagulation and thrombosis? Front Oncol 2: 115, 2012.

37. Zhang Q, Bai X, Chen W, Ma T, Hu Q, Liang C, Xie S, Chen $\mathrm{C}, \mathrm{Hu} \mathrm{L}, \mathrm{Xu} \mathrm{S}$ and Liang T: Wnt/ $\beta$-catenin signaling enhances hypoxia-induced epithelial-mesenchymal transition in hepatocellular carcinoma via crosstalk with hif-1 $\alpha$ signaling. Carcinogenesis 34: 962-973, 2013.

38. Misra A, Pandey C, Sze SK and Thanabalu T: Hypoxia activated EGFR signaling induces epithelial to mesenchymal transition (EMT). PLoS One 7: e49766, 2012.

39. Matsuoka J, Yashiro M, Doi Y, Fuyuhiro Y, Kato Y, Shinto O, Noda S, Kashiwagi S, Aomatsu N, Hirakawa T, et al: Hypoxia stimulates the EMT of gastric cancer cells through autocrine TGF $\beta$ signaling. PLoS One 8: e62310, 2013.

40. Salnikov AV, Liu L, Platen M, Gladkich J, Salnikova O, Ryschich E, Mattern J, Moldenhauer G, Werner J, Schemmer P, et al: Hypoxia induces EMT in low and highly aggressive pancreatic tumor cells but only cells with cancer stem cell characteristics acquire pronounced migratory potential. PLoS One 7: e46391, 2012.

41. Cannito S, Novo E, Compagnone A, Valfrè di Bonzo L, Busletta C, Zamara E, Paternostro C, Povero D, Bandino A, Bozzo F, et al: Redox mechanisms switch on hypoxia-dependent epithelial-mesenchymal transition in cancer cells. Carcinogenesis 29: 2267-2278, 2008.

42. Hong Y and Zhang Q: Phenotype of circulating tumor cells: Face-off between epithelial and mesenchymal masks. Tumor Biol 37: 5663-5674, 2016.

43. Pierga JY, Hajage D, Bachelot T, et al: High independent prognostic and predictive value of circulating tumor cells compared with serum tumor markers in a large prospective trial in first-line chemotherapy for metastatic breast cancer patients. Ann Oncol 23: 618-624, 2012.

44. Giuliano M, Giordano A, Jackson S, et al: Circulating tumor cells as prognostic and predictive markers in metastatic breast cancer patients receiving first-line systemic treatment. Breast Cancer Res 13: R67, 2011.

45. de Bono JS, Scher HI, Montgomery RB, Parker C, Miller MC, Tissing H, Doyle GV, Terstappen LW, Pienta KJ and Raghavan D: Circulating tumor cells predict survival benefit from treatment in metastatic castration-resistant prostate cancer. Clin Cancer Res 14: 6302-6309, 2008.

46. Schulze K, Gasch C, Staufer K, Nashan B, Lohse AW, Pantel K, Riethdorf S and Wege H: Presence of EpCAM-positive circulating tumor cells as biomarker for systemic disease strongly correlates to survival in patients with hepatocellular carcinoma. Int J Cancer 133: 2165-2171, 2013.

47. Allard WJ, Matera J, Miller MC, Repollet M, Connelly MC, Rao C, Tibbe AG, Uhr JW and Terstappen LW: Tumor cells circulate in the peripheral blood of all major carcinomas but not in healthy subjects or patients with nonmalignant diseases. Clin Cancer Res 10: 6897-6904, 2004.

48. Aceto N, Bardia A, Miyamoto DT, Donaldson MC, Wittner BS, Spencer JA, Yu M, Pely A, Engstrom A, Zhu H, et al: Circulating tumor cell clusters are oligoclonal precursors of breast cancer metastasis. Cell 158: 1110-1122, 2014.

49. Visvader JE and Lindeman GJ: Cancer stem cells in solid tumours: Accumulating evidence and unresolved questions. Nat Rev Cancer 8: 755-768, 2008.

50. Tinhofer I, Saki M, Niehr F, Keilholz U and Budach V: Cancer stem cell characteristics of circulating tumor cells. Int J Radiat Biol 90: 622-627, 2014.

51. Swann JB and Smyth MJ: Immune surveillance of tumors. J Clin Invest 117: 1137-1146, 2007.

52. Stott SL, Hsu CH, Tsukrov DI, Yu M, Miyamoto DT, Waltman BA, Rothenberg SM, Shah AM, Smas ME, Korir GK, et al: Isolation of circulating tumor cells using a microvortex-generating herringbone-chip. Proc Natl Acad Sci USA 107: 18392-18397, 2010

53. Hosokawa M, Yoshikawa T, Negishi R, Yoshino T, Koh Y, Kenmotsu H, Naito T, Takahashi T, Yamamoto N, Kikuhara Y, et al: Microcavity array system for size-based enrichment of circulating tumor cells from the blood of patients with small-cell lung cancer. Anal Chem 85: 5692-5698, 2013

54. Sollier E, Go DE, Che J, et al: Size-selective collection of circulating tumor cells using Vortex technology. Lab Chip 14: 63-77, 2014. 